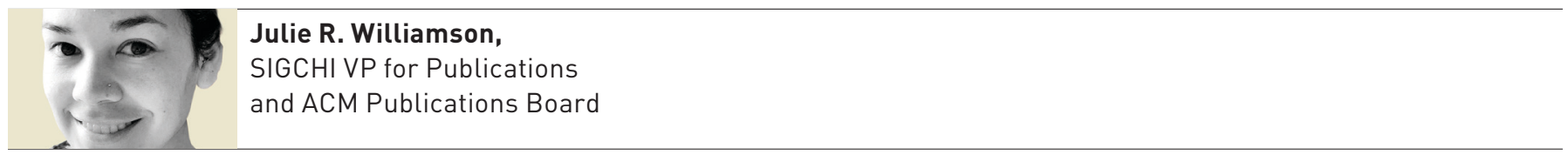

\title{
Publication Matters
}

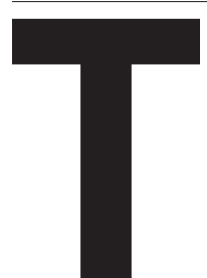

he way that ACM

archives publications is changing. Although our working practice as authors, reviewers, and readers must adapt to accommodate these changes, the benefits of a more resilient, flexible, and accessible archive are worth such an effort.

Separating content from presentation. The key motivation for changing our publication process is to archive publications as markup, not PDF. Archiving the content of publications in a raw format that is independent of the presentation is a significant step forward. This approach adds new capabilities to our ACM Digital Library and better prepares us for the future.

Separating content from presentation means we can view publications in dynamic formats, including accessible HTML, responsive layouts in browsers and e-readers, and new formats we haven't yet imagined. Decoupling our work from PDF also enables a more resilient archive, preparing us for a future that moves away from print formats and print format metaphors.

The new publication workflow. ACM has developed a new publication platform called the ACM Publication System (TAPS) [1]. TAPS automates some of the manual work completed by proceedings editors, such as checking for formatting and required sections, and adds new functionality to the publication production process.

TAPS takes a single column source file in LaTex or Word and converts this into a two-column PDF, responsive HTML, and ePub formats. Authors then iterate on these documents using

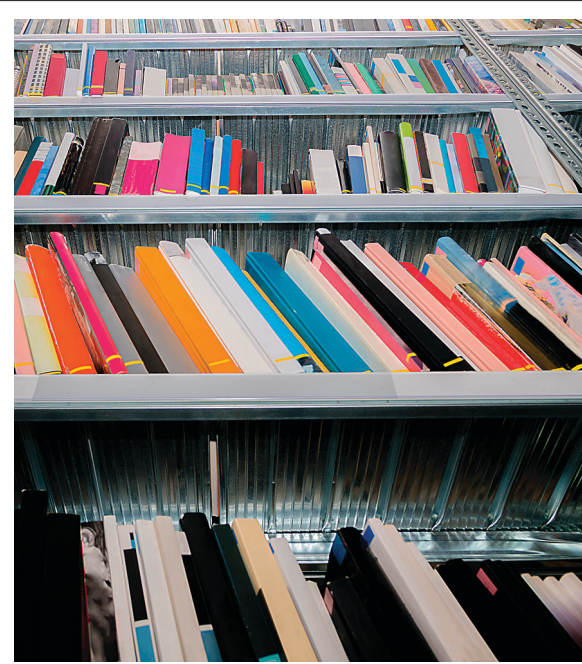

TAPS, correcting any issues and ensuring their publications look and feel exactly how they want. This interaction with TAPS replaces asynchronous email communication with a proceedings vendor.

Once authors are happy with their final versions, these documents are ready for archiving in the ACM Digital Library. Conferences are already producing proceedings using TAPS, including TVX 2019 [2], ICMI 2019 [3], and WWW 2019 [4].

Changes for authors. As we transition to the new ACM workflow, you can expect to see changes in how you prepare and review publications. The most visible change is submitting your manuscript in a single column format, limited by words or characters instead of pages. Submission documents will include minimal formatting, as required for compatibility with TAPS, and encourages authors and reviewers to focus on content, not layout.

There are also some additional steps that Word authors will complete to prepare their accepted submissions for publication. This includes adding markup to their references and validating the structure and application of styles in their document [5].

SIGCHI support for authors. SIGCHI volunteers have been actively involved in testing TAPS, developing usable templates, and exploring how TAPS can be incorporated into our current publication practices. SIGCHI volunteers are also engaged in policy development at ACM, for example, contributing to policies on conflict of interest and name changes in the ACM Digital Library.

The SIGCHI Executive Committee (EC) supports authors through resources on the SIGCHI website. For example, there are numerous policies and procedures around conduct, ethics, and harassment, and the EC has summarized these policies to help authors navigate these complicated issues [6].

If you are impacted by ACM or SIGCHI policies, the SIGCHI EC can advise and/or advocate for authors. For more information, please contact the EC at sigchi-ec@acm.org.

\section{ENDNOTES}

1. https://www.acm.org/publications/taps/ word-template-workflow

2. https://dl.acm.org/citation. cfm?id=3317697

3. https://dl.acm.org/citation. cfm?id $=3340555$

4. https://dl.acm.org/citation. cfm?id=3308558

5. https://youtu.be/sUGEoPaXRKQ

6. https://sigchi.org/ethics/

(1) Julie R. Williamson, lecturer in humancomputer interaction at the University of Glasgow, is SIGCHI VP for Publications and a member of the ACM Publications Board.

$\rightarrow$ Julie.Williamsonaglasgow.ac.uk

$\rightarrow$ aJRicoWilliamson 